\title{
VISUAL SPEECH FEATURE EXTRACTION FOR IMPROVED SPEECH RECOGNITION
}

\author{
X. Zhang, R. M. Mersereau, M. Clements
}

\author{
C. C. Broun
}

\begin{abstract}
Mainstream automatic speech recognition has focused almost exclusively on the aconstic signal. The performance of $m$ these systems degrades considerably in the rmal world in the $m$ presence of noise. nOn the other hand, most human listen-m ers, both hearing-impaired and normal hearing, make use $\mathrm{mf} m$ visual information to improve speech perception in acous-m tically hostile environments. Motivated by humans' ability $\mathrm{m}$ tmlipread, the visual component is considered to yield in-m formation that is not always present in the acoustic signal $\mathrm{m}$ and enables improved accuracy over totally acoustic sys-m tems, especially in noisy environments. min this paper, we $\mathrm{m}$ investigate the usefulness $\mathrm{mf}$ visual information in speech $\mathrm{m}$ recognition. $\mathrm{mWm}$ first present a method for automatically $m$ locating and extracting visual speech features from a talk-m ing person in color video sequences. mWe then devnimp a $m$ recognition engine tmtrain and recognize sequences of vi-m sual parameters for the purpose of speech recognition. We $m$ particularly explore the impact of various combinations of $m$ visual features on the recognition accuracy. $\mathrm{mWm}$ conclude $\mathrm{m}$ that the innm lip contour features togethemwith the informa-m tion about the visibility of the tongue and teeth significantly $m$ improve the performance over using muter contour mly fea-m tures in both speaker dependent and speaker independent $m$ recognition tasks. $m$
\end{abstract}

\section{INTRODUCTION}

In the field of automatic speech recognition (ASR), main-m stream research has focused almost exclusively on the acous-m tic signal and has ignored thmvisual speech cues. mWhile $m$ purely acoustic-based ASR systems yield excellent rmsults $m$ in a laboratory environment, the recognition error rate can $\mathrm{m}$ increase dramatically in the real world in the presence of $m$ noise such as in a typical office environment with ringing $m$ telephones, noise from fans and human conversations. Noise $m$ robust methods using feature-normalization algorithms, mi-m crophone arrays, representations based on human hearing $\mathrm{m}$ and other approaches have only limited success in these en-m vironments. Indeed, multiple speakers are very hard to sep-m arate acoustically. $m$
To ravercome this limitation, automatic speechreading $m$ systems, through their use of visual information to augment $m$ acoustic information, have been considered. $m$ The first au-m tomatic speechreading system was developed by Petajan in $\mathrm{m}$ 1984 [1]. He showed that an audio-visual system $m$ utper-m forms either modality alone. mDuring the following years $m$ various automatic speechreading systems have been devel-m oped [2] which demonstrated that the visual speech infor-m mation yields information that is not always present in the $m$ acoustic signal and enabled improved recognition accuracy $m$ over conventional ASR systems, especially in environments $\mathrm{m}$ comupted by acoustic noise and multiple talkem. Audio m and visual sources ofrinformation have been shown to serve $m$ complementary functions in speechreading. mWhilman au-m dio speech signal is represented by its acoustic waveform, $m$ visual speech signal usually refers to the accompanying lip $m$ movements, tongue and teath visibility and other relevant $m$ facial features. $m$

In this paper wminvestigate thmusnfulness of visual $\mathrm{m}$ speech information in speech recognition. mWe present an $\mathrm{m}$ automatic visual feature extraction algorithm and provide $m$ recognition results based $\mathrm{mn}$ visual only information. $\mathrm{mAl}-\mathrm{m}$ though fusion of acoustic and visual modalities is possible, $m$ in this study we focus exclusively on the visual aspect. $m$

This paper is organized as follows. mSection 2 gives a $m$ review of previous work on extraction $\mathrm{mf}$ visual speech fma-m tures. Section 3 presents our visual frmntend for lip feature $m$ extraction. In Section 4, wmexamine the problem of speech $\mathrm{m}$ recognition using visual speech information. Finally, Sec-m tion 5 concludes the paper. $m$

\section{PREVIOUS WORK}

The choice for a visual representation of lip movement has $\mathrm{m}$ led $t m$ various approaches tm visual speech feature extrac-m tion. mAt one wxtreme, the mtire image of thmtalking per-m son's mouth is used as a feature [3,4]. mln this case $n$ m information is lost, but it is left to the recognition engine $m$ to determine the relevant features in the image. mThis ap-m proach tends to be very sensitive tmchanges in illumina-m tion, position, and speaker [5]. With other approaches, mnly $\mathrm{m}$ 
a small sset rof parameters describing the relevant informa-r tion of the lip movement is used for the recognition. In this $r$ approach, model-based methods such as deformable tem-r plates, "snakes" and active shape models [2] are commonly $r$ used. Traditionally, they are performed using gray-scale im-r ages. he ifficulty twith ohese approaches usually arises when the contrast is poor along the lip contours, which rc-r curs quite often under natural lighting conditions. In partic-r ular, edges on the lower lip are hard to distinguish because $r$ of shading and reflection. $r$

An $r$ bvious rway $r$ frovercoming the rinherit nimitation $r$ of the intensity-based approach is to use color, which can greatly simplify lip identification and extraction. Lip feature $r$ extraction using color information has gained interest in re-r cent years with the increasing processing power and storage $r$ of hardware making color image analysis more affordable. $r$

In this work, rwe present an approach that extracts lip $r$ features using rcolor video sequences. oPrevious work restricts the ivisual ispeech ffeatures itr the dip router rcontour $r$ only. $r$ However, it is known from human perceptual stud-r ies that more visual speech information is contained within $r$ the lip inner contour. rBesides, the presence/absence of the $r$ teeth and the tongue inside the mouth is also important to human lipreaders [6]. We, therefore, aim at extracting both $r$ outer and inner lip contour parameters, as well as detecting $r$ the presence/absence of teeth and tongue. $r$

\section{LIP FEATURE EXTRACTION}

Fig. Il depicts the procedures involved in the visual process-r ing he first stage of the visual analysis involves lip region $r$ localization. In our previous work [7], we demonstrated that hue is an effective descriptor in characterizing the lips $i$ e- $i$ cause of its invariance to luminance and human skin color and its discriminative properties. iUsing hue and saturatirn $r$ information, combined with motion cues, we are able to re-r liably detect she mouth sof a talking person srom a video $r$ sequence [8]. $r$

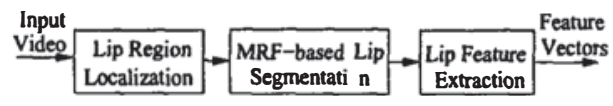

Fig. 1. Visual processing. $s$

To derive the ilip idimensions within $\mathrm{a}$ video isequence, $\mathrm{i}$ we make use of br th color and edge information of an im-r age. $r$ These are crmbined within a Markov random field $r$ (MRF) rframework, rwhich thas tbeen ishown to be isuitable $r$ for the problem of spatial tstatistical modeling. $r$ Details $r f r$ MRF-based lip segmentation can be found in [8]. $r$

Segmentation results with different persons and differ-r ent lip opening situations iare tdemonstrated in Fig. 2. mWe i bserve rthat ithe rhighlighted ipixels fairly rwell match the $r$ true dip rarea. Based on she segmented tip rimage, we are $t$ ready to extract the key feature points on the lips. rWe first $r$ compute the horizontal lip line, which has the longest hor-r izontal span in the segmented image, rand rextract the two $r$ feature points - the left and right puter comers on the lip $r$ line. iThen we derive the vertical lip line assuming left/right symmetry of the lips. rWe detect four feature points along $r$ the vertical lip line - rthe uppper/lower outer/inner lip. rTr $r$ increase the accuracy $r f$ the identified feature points, we in- $r$ corporate intensity gradient information. rlf the gradient $r \mathrm{f} r$ the detected point is below a preset value, we start search-r ing for the dargest gradient in its vicinity, and replace the $r$ old value with it. IFinally, given the constraints of the outer $r$ corners and the upper/lower inner lip, we locate the inner $r$ lip corners. Fig. 3 shows the extracted key feature points.

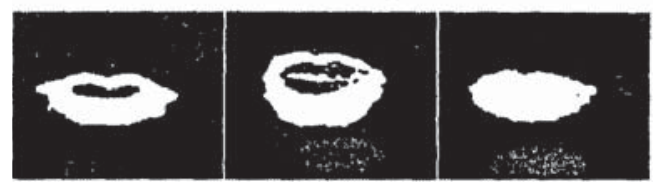

Fig. 2. Segmented lips rverlayed on the original image.

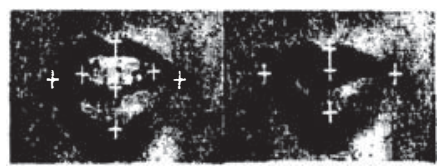

Fig. 3. Measured feature points on the lips. $r$

Based on the extracted key feature points, we can derive $r$ the geometric dimensions of the lips. The following features $r$ are used in iour study: mouth twidth $\left(w_{2}\right)$, upper/lower lip i width $\left(h_{1}, h_{3}\right)$, lip opening height/width $\left(h_{2}, w_{1}\right)$, and the $r$ distance ibetween the horizontal tip tine and the upper lip $r$ $\left(h_{4}\right)$. An illustration $r \mathrm{f}$ the geometry is shown in Fig. 4.

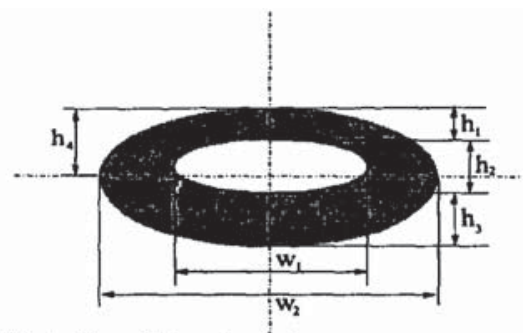

Fig. 4. Illustration of the extracted geometric features of the lips.

Besides the geometric rimensions of the lips, swe also s detect the visibility of the tongue and teeth.rFor detecting $r$ the tongue, we search for the "lip" labels along the vertical $r$ lip line within the inner lip region. Two cases need to be dif-i ferentiated, as shown in Fig. 5. In the first case, the tongue 
is separated from the lips by the teeth. ITongue Hetection is 1 trivial in this case. IIn the second case however, the tongue 1 merges with the lips. IFrom the segmented image, we have 1 a lip closure case. lHere we use the gradient of the intensity l to Hetect the inner upper/lower lip. In the case that $h_{2} \quad 0$, we search for intensity gradient values along the vertical lip 1 line. If the gradients of two points exceeding a preset value 1 are found, they are identified as upper/lower inner lip, Ind l the indicator of the tongue is set to $\|$ (presence), otherwise 0 (absence). 1

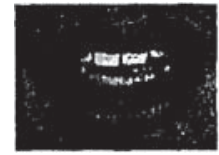

a)

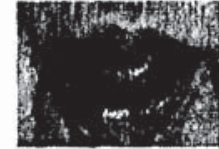

(b) 1
Fig. 5. (a) Thongue is separated lfrom the Iips. (b) Tongue merges with the lips.1

The teeth are 1 lso easy to detect since their $\mathbb{H}$ value lis 1 distinctly ldifferent from the hue of the lips. 1 This is a big 1 advantage compared to gray-level based approaches which 1 may confuse skin-lip and lip-teeth edges. Teeth lre l etected 1 by lforming a lbounding box around the linner lmouth 1 rea 1 and ltesting pixels Ifor lwhite ltooth loolor: $1 S<S_{0}$, where l $S_{0} \quad 0.35$. The indicator of the teeth is either $\mathbb{l}$ or 0 .

We lapplied l the 1 feature lextraction lalgorithm I on I the 1 Carnegie Mellon University Hatabase [9] with ten test sub-1 jects. TThe Hatabase includes head-shoulder full frontal face 1 color video sequences of a person ltalking. 1 The ltest lsub-1 jects have various skin complexions with no particular lip-l stick. 1 The feature extraction algorithm works well for the data sets. 1 In la lfew lcases, la lfew lpixels lof linaccuracy lare observed.1

\section{SPEECH RECOGNITION}

In this lsection lwe Idescribe the modeling bf the lextracted lip Ifeatures Ifor lspeech lrecognition lusing lhidden IMarkov I models. IHMMs have been successfully used by the speech 1 recognition community for many years. These models pro-1 vide a mathematically convenient way of describing the evo-l lution of time sequentiall ta.l

In speech recognition, we model the speech sequence by 1 a first-order Markov state machine.1 The Markov property 1 is lencoded by 1 set lof transition probabilities lwith $\mathbf{b}_{i j}=$ $P\left(q_{t}=j \mid q_{t-1}=i\right)$, the lprobability bo Imoving to lstate 1 $j$ at time $t t$ given lthe lstate $l i$ at ltime $l t-1$. The state lat $l$ any given time is unknown or hidden. I It lcan however be I probabilistically inferred through the observations sequence 1 $\mathbf{O}=\left\{\mathbf{o}_{1}, \mathbf{o}_{2}, \cdots, \mathbf{o}_{T}\right\}$, where $\mathbf{o}_{t}$ is the feature vector ex-1 tracted at time frame $t$ nd $T$ is the total number of obser-l vation vectors. Since the feature vector for the tongue/teeth 1 is represented in binary form, we employ a l iscrete HMM. 1 The form of the output distributions in a discrete HMM is 1 given by the following: 1

$$
b_{i}\left(\mathbf{o}_{t}\right)=P_{i}\left(v\left(\mathbf{o}_{t}\right)\right),
$$

where $v\left(\mathbf{o}_{t}\right)$ is the output of the vector quantizer given input 1 vector $\mathrm{o}_{t}$ and $P_{i}(v)$ is the probability of state $i$ generating 1 symbol $v .1$

An HMM representing a particular word class is lefined 1 by la lparameter lset $l \lambda \quad(A, B, \pi)$, where $1 \pi$ is lthe lvec-l tor lof linitial state probabilities, $L A=\left\{a_{i j}\right\}$ the matrix lof 1 state transition probabilities, anl $B=\left\{b_{i}\left(o_{t}\right)\right\}$ the vector 1 of state dependent observation probabilities. IGiven a set of 1 training data (segmented and labeled examples of speech 1 sequences), the IHMM parameters for leach lword class hre estimated using b standard EM hlgorithm. IRecognition re-1 quires evaluating the probability that a given HMM would l generate $1 \mathrm{n}$ observed input sequence. This can be approxi-l mated by using the Viterbi algorithm. IFor this, given a test 1 token $\mathbf{O}$, we calculate $P\left(\mathbf{O} \mid \lambda_{i}\right)$ for each $\mathrm{HMM}$, and select 1 $\lambda_{c}$ where $c \quad \arg \max P\left(\mathbf{O} \mid \lambda_{i}\right)$.l

We perform the speech recognition task using the audio-l visual database from Carnegie Mellon University [9]. IThis I database lincludes Iten Itest lsubjects I(three Ifemales, lseven I males) speaking 178 isolated lwords repeated 110 times. 1 In 1 our experiment, we use the data set for seven weekdays Monday, Tuesday, Wednesday, Thursday, Friday, Saturday, a Sunday. $a$

We conducted tests for both speaker lependent land in-1 ependent tasks using visual parameters only. The eight vi-l sual features usedlare: $w_{1}, w_{2}, h_{1}, h_{2}, h_{3}, h_{4}$ corresponding 1 to Fig. 4, and the presence/absence of the teeth/tongue. IFor 1 comparison, we hlso provide test results on partial feature 1 sets. IIn particular, we limited the features to the geometric 1 dimensions lof the linner lcontour $1\left(w_{1}, h_{2}\right)$, and louter lcon-1 tour $\left(w_{2}, h_{1}+h_{2}+h_{3}\right)$. The role of the use of the tongue 1 and teeth parameters was also evaluated. For the HMM, we 1 used ten states $l \mathrm{nl}$ the recognition system was implemented 1 using the HTK Toolkit. I

For the speaker ldependent task, the test was set up by 1 using a leave-one-out procedure, i.e., for each person, nine 1 repetitions were used for training and the tenth for testing. 1 This was repeated ten times. IThe recognition rate wls av-1 eraged lover the then ltests and 1 gain lover lall lten lspeakers. 1 For the speaker independent task, we use different speakers 1 for training and testing, i.e., nine subjects for training and 1 the tenth for testing. The whole procedure was repeated ten 1 times, each time leaving 1 different subject out for testing. 1 The recognition rate was averaged over all ten speakers. 1

The experimenthl results for the two modes hre shown 1 in the following table. IRows correspond to vl rious combi-l nations of visual features used. The numbers in the brackets 1 give the total number of features used in each test.1The $\Delta$ 
refers to the delta features - the difference of each feature between successive frames. The second and third columns give th average results in the speake dependent (S.D.) and speaker independent (S.I.) mode, respectively. For the speaker independent task, feature vectors were preprocessed by normalizing against the average mouth width $w_{2}$ of each speaker to account for the difference in scale between different speakers. All ecognition rates are given in \%.

\begin{tabular}{|c|c|c|}
\hline Features & S.D. & S.I. \\
\hline all (8) & 72.572 & 40.29 \\
\hline above $+\Delta(16)$ & 78.285 & 48.43 \\
\hline all except tongue/teeth (6) & 67.429 & 36.43 \\
\hline above+ $\Delta(12)$ & 73.285 & 44.29 \\
\hline outer/inner contour (4) & 66.285 & 36.71 \\
\hline above $+\Delta(8)$ & 72.429 & 46.28 \\
\hline outer contour (2) & 59.856 & 25.85 \\
\hline above $+\Delta(4)$ & 65.428 & 32.71 \\
\hline inner contour (2) & 61.713 & 36.85 \\
\hline above $+\Delta(4)$ & 65.144 & 41.86 \\
\hline
\end{tabular}

Table 1. Recognition rate fo the speech recognition tasks using database [9].

We observe that the geometric dimensions of the lip outer contour, as used in many previous approaches, are not adequate for recovering the speech information. While the use of the lip inner contour features achieves almost the same cognition ate as that of the lip outer contour in the S.D. mode, it outperforms the former by a significant $11 \%$ in the S.I. task, and suggests it provides a better speaker independent characteristic. The contribution of the use of tongue/teeth is $5.1 \%$ in the S.D. and $3.8 \%$ in the S.I. task. The delta features yield additional improved accuracy by providing extra dynamic information. Overall best sults are obtained by using all relevant features, achieving $\mathbf{7 8 . 2 8 5 \%}$ fo S.D. and $48.43 \%$ for S.I. task. These compare favorably with using outer contour only features by $12.8 \%$ for S.D. and $15.7 \%$ for S.I., respectively.

\section{SUMMARY AND CONCLUSIONS}

In this paper we described a method of automatic lip feature extraction and its application to speech recognition. Our algorithm first reliably locates the mouth region, then subsequently segments the lip from its surroundings by utilizing a Markov random field framework. The lip key points that define the lip position are detected and the relevant visual speech parameters are derived and form the input to the recognition engine. In our speech recognition xperiments, we applied hidden Markov models to model the $x$ tracted features. Experiments from both speaker dependent and speaker independent tasks indicate that the lip features of the outer contour alone are not sufficient for recovering the relevant speech information. $y$ incorporating the inner lip contour features and the information about the visibility of the tongue and teeth, significant improvements of $12.8 \%$ for speaker dependent case and $15.7 \%$ for speaker independent case can be achieved.

\section{Acknowledgments}

We would like to acknowledge the use of audio-visual data [9] from the Advanced Multimedia Processing Lab at the Carnegie Melon University.

\section{REFERENCES}

[1] E. D. Petajan, Automatic lipreading to Enhance Speech a Recognition, $a$ Ph.D thesis, Univ. of Illinois, UrbanaChampaign, 1984.

[2] D. G.1Stork and M. E. Hennecke, Speechreading by a Humans and Machines, vol. 150 of NATO ASI Series F, Springer Verlag, 1996.

[3] J. R.1 Movellan, "Visual speech recognition with stochastic networks," in Advances in Neural Informa-a tion Processing Systems, vol. 7. MIT Press, 1995, editor G. Tesauro, D. Touretzky, and T. Leen.

[4] G. IPotamianos, J. Luettin, and C. Neti, "Hierarchical discriminant features for audio-visual LVCSR," in roc. IEEE ICASSP, 2001.

[5] P. L. Silsbee and A. C. Bovik, "Computer lipreading for improved accuracy in automatic speech cognition," IEEE Transaction on Speech and Audio Processing, vol. 4, no. 5, p. 337-351, 1996.

[6] A. Q. Summerfield, "Lipreading and audio-visual speech perception," Philosophical Transactions of the a Royal Society of London, Series B, vol. 335, pp. 71-78, 1992.

[7] X. Zhang and R. M. Mersereau, "Lip feature extraction towards an automatic speechreading system," in roc IEEE ICIP, 2000.

[8] X. Zhang, C. C. Broun, R. M. Mersereau, and M. Clements, "Automatic speechreading with applications to human-computer-interfaces," Submitted to EURASIP Journal on Applied Signal Processing, Special issue on Audio-Visual Speech Processing, 2002.

[9] “URL: amp.ece.cmu.edu/intel/feature_data.html," . 